\title{
OPERATORS COMMUTING WITH BOOLEAN ALGEBRAS OF PROJECTIONS OF INFINITE MULTIPLICITY
}

\author{
BY \\ L. TZAFRIRI $\left({ }^{1}\right)$
}

The algebra of operators commuting with a Boolean algebra of projections of finite uniform multiplicity (in the sense of Bade [3]) has been studied by Foguel [10] and the author [12]. The aim of the present paper is to show that most properties described in these two papers can be extended (sometimes, under additional conditions) to the algebra $\Xi$ of all the operators which commute with a complete countably decomposable Boolean algebra of projections containing no projections of infinite uniform multiplicity. It should be mentioned that one cannot get interesting general results in the case in which there are projections of infinite uniform multiplicity in the Boolean algebra of projections, since every operator on a Banach space commutes with the Boolean algebra of projections composed from the identities 0 and $I$.

We shall start by proving that for every operator $A \in \Xi$ there is a sequence of projections belonging to the above mentioned Boolean algebra of projections which increases to the identity and $A$ multiplied by any element of this sequence is a spectral operator. Relying on this result we study the spectrum of operators of $\Xi$ and give a necessary and sufficient condition for such an operator to be spectral.

In the following section we generalize Theorem 8 of [12] showing that in $\Xi$ a strong limit of spectral operators on a Hilbert space is spectral provided that they are of the same finite type and their resolutions of the identity are uniformly bounded. Adequate examples elucidate why we require the boundedness of the type of the spectral operators in most theorems.

1. Preliminaries. For convenience we shall summarize here some definitions and results concerning the Bade theory of multiplicity for Boolean algebras of projections on Banach spaces (see [2] and [3] and the general theory of spectral operators (see [4], [6], [7], [8], and [13])).

According to Bade [3], a Boolean algebra (B.A.) of projections $\mathscr{E}$ will be called complete if for every family $\left\{E_{\alpha}\right\} \subseteq \mathscr{E}$ the projections $\bigvee E_{\alpha}$ and $\bigwedge E_{\alpha}$ exist in $\mathscr{E}$ and, moreover

$$
\left(\bigvee E_{\alpha}\right) X=\operatorname{clm}\left\{E_{\alpha} X\right\} ; \quad\left(\bigwedge E_{\alpha}\right) X=\bigcap E_{\alpha} X
$$

Received by the editors October 22, 1965 and, in revised form, October 25, 1966.

( $\left.{ }^{1}\right)$ This paper is part of the author's Ph.D. thesis to be submitted to the Hebrew University. The author wishes to express his gratitude to Professor S. R. Foguel for his guidance and kind encouragement. 
A projection $E \in \mathscr{E}$ will be called countably decomposable if every family of disjoint projections in $\mathscr{E}$ bounded by $E$ is at most countable. If the identity $I \in \mathscr{E}$ satisfies this condition $\mathscr{E}$ will be called countably decomposable.

A function $m$ on a complete abstract B.A. $\mathscr{E}$ whose values are cardinals will be called a multiplicity function provided

$$
m(0)=0 \quad \text { and } \quad m\left(\bigvee E_{\alpha}\right)=\bigvee_{\alpha} m\left(E_{\alpha}\right) ; \quad\left\{E_{\alpha}\right\} \subseteq \mathscr{E}
$$

The cardinal number $m(E)$ is called the multiplicity of $E$. We say that $E \in \mathscr{E}$ has uniform multiplicity $p$ if $m(F)=p$ whenever $0 \neq F \leqq E$.

The cyclic subspace spanned by a vector $x$ is defined by

$$
\mathfrak{M}(x)=\operatorname{cl} \mathrm{m}\{E x \mid E \in \mathscr{E}\} .
$$

Then, the following theorem (due to Bade) holds:

THEOREM A. Let $\mathscr{E}$ be a complete B.A. of projections in a Banach space X. There exists a unique multiplicity function $m$ defined on $\mathscr{E}$ with the property that for each $E \in \mathscr{E}$ which is countably decomposable, $m(E)$ is the least cardinal power of a set of cyclic subspaces spanning the range of $E$. There is a unique decomposition of the identity

$$
I=\bigvee E_{v}
$$

into disjoint projections such that if $E_{v} \neq 0, E_{v}$ has uniform multiplicity $\nu$.

According to [5, p. 580], for any bounded linear operator $A, \rho(A)$ will denote its resolvent set, $\sigma(A)$ its spectrum, $\sigma_{p}(A)$ its point spectrum, $\sigma_{c}(A)$ its continuous spectrum and $\sigma_{r}(A)$ its residual one. For $\lambda \in \rho(A)$ we shall write $R(\lambda, A)=(\lambda I-A)^{-1}$.

The next two results were proved by Dunford [4, Lemma 3] and [6, Lemma 1].

THEOREM B. Let $E$ be the resolution of the identity for the spectral operator A, and let $N$ be its generalized nilpotent part. Then in the uniform operator topology, and uniformly with respect to $\xi$ in any closed set $\rho \subset \rho(A)$, we have

$$
R(\xi, A)=\sum_{\nu=0}^{\infty} N^{v} \int \frac{E(d \lambda)}{(\xi-\lambda)^{v+1}}
$$

THEOREM C. Let $E_{1}, \ldots, E_{v}$ be bounded disjoint projections in $X$, each commuting with the bounded operator $A$ and such that $I=E_{1}+\cdots+E_{v}$. Then $A$ is a spectral operator if and only if each restriction $A / E_{i} X$ is a spectral operator. If $A$ is a spectral operator, then the resolution of the identity for the restriction $A \mid E_{i} X$ is the corresponding restriction of the resolution of the identity for $A$.

The commuting spectral measures on Hilbert spaces were first studied by Wermer [13]. We shall quote this remarkable result. 
THEOREM D. Let $E(\cdot)$ and $F(\cdot)$ be two commuting spectral measures on Hilbert space whose bounds are $M$, respectively $L$. Then, the Boolean algebra of projections generated by $E(\cdot)$ and $F(\cdot)$ is bounded by $4 M^{2} L$.

Let $A$ be a spectral operator whose resolution of the identity is $E(\cdot)$. By Foguel [8, Lemma 1, p. 59], $A$ has a unique decomposition

$$
A=\int(\operatorname{Re} \lambda) E(d \lambda)+i \int(\operatorname{Im} \lambda) E(d \lambda)+N
$$

where $N$ is the generalized nilpotent part and $R=\operatorname{Re} A=\int(\operatorname{Re} \lambda) E(d \lambda)$ and $J=\operatorname{Im} A=\int(\operatorname{Im} \lambda) E(d \lambda)$ are called the real, respectively imaginary part of $A$.

We shall conclude this section by quoting the following result proved in [12, Theorem 8].

THEOREM E. Let $\left\{A_{n}\right\}$ be a sequence of spectral operators commuting with a B.A. of projections of finite uniform multiplicity $N$ and assume that it converges strongly to an operator $A$. Let $G\left(A_{n}, \cdot\right)$ denote the resolution of the identity for $A_{n}$. If there is a constant $L$ such that $\left\|G\left(A_{n}, \delta\right)\right\| \leqq L ; n=1,2, \ldots, \delta \varepsilon$ Borel sets, then $A$ is spectral (of type $N$ ). Moreover, the sequence of the scalar parts of $A$ converges strongly to the scalar part of $A$ and the same assertion holds with respect to the nilpotent parts, real parts and imaginary parts.

2. Notation. Throughout the paper $X$ denotes a fixed Banach space unless otherwise specified, $\mathscr{E}$ is a complete, countably decomposable B.A. of projections containing no projections of infinite uniform multiplicity and $\Xi$ the algebra of all the operators which commute with $\mathscr{E}$.

3. Spectral properties. Foguel has proved in [10, Theorem 2.3] that for every operator $A$ commuting with a complete countably decomposable B.A. of projections of finite uniform multiplicity there is a sequence of projections belonging to the mentioned B.A. which increases to the identity and such that $A$ multiplied by any projection of this sequence is spectral. It will be shown now that this property still holds in our case.

LEMMA 1. Assume $\mathscr{B}$ is a complete countably decomposable B.A. of projections of finite multiplicity $p$ (not necessarily uniform) and $A$ an operator commuting with $\mathscr{B}$. Then, there exists an increasing sequence $\left\{F_{n}\right\} ; F_{n} \in \mathscr{B} ; n=1,2, \ldots$ such that $\lim _{n \rightarrow \infty} F_{n} x=x ; x \in X$ and $A F_{n} ; n=1,2, \ldots$ are spectral operators of finite type $p$.

Proof. By (1.4) $I=\bigvee_{i=1}^{p} E_{i}=\sum_{i=1}^{p} E_{i}$ where $E_{i}=0$ or it has uniform multiplicity $i$ and the projections $E_{i}, 1 \leqq i \leqq p$, are disjoint. Then $A=\sum_{i=1}^{p} A E_{i}$ and the operator $A / E_{i} X$ (the restriction of $A$ to $E_{i} X$ ) commutes with the complete countably decomposable B.A. of projections

$$
\mathscr{B}_{i}=\left\{E / E_{i} X \mid E \in \mathscr{B}\right\} .
$$


Since $\mathscr{B}_{i}$ has finite uniform multiplicity $i$, in view of [10, Theorem 2.3], the statement of the lemma holds for $A \mid E_{i} X, 1 \leqq i \leqq p$, and consequently for $A$.

Theorem 2. Let $A \in \Xi$. There exists an increasing sequence $\left\{P_{k}\right\}, P_{k} \in \mathscr{E}$, $k=1,2, \ldots$ which converges strongly to the identity I and such that

(i) $P_{k}$ has finite multiplicity.

(ii) $A P_{k}$ is a spectral operator of finite type.

Proof. According to (1.4) and our hypotheses we get

$$
I=\bigvee_{n=1}^{\infty} E_{n}
$$

where $E_{n}=0$ or $E_{n}$ has uniform multiplicity $n, n=1,2, \ldots$ Put

$$
G_{p}=\bigvee_{n=1}^{p} E_{n}, \quad p=1,2, \ldots
$$

One can easily see that $G_{p} \in \mathscr{E}$ and it has finite multiplicity. Moreover $G_{1} \leqq G_{2}$ $\leqq \cdots \leqq G_{p} \leqq \cdots$ and $I=\bigvee_{p=1}^{\infty} G_{p}$. Thus, by Bade [2, Lemma 2.3], $\left\{G_{p}\right\}$ converges strongly to $I$. Denote

$$
\mathscr{E}_{p}=\left\{E / G_{p} X \mid E \in \mathscr{E}\right\}, \quad p=1,2, \ldots
$$

It is a complete countably decomposable B.A. of projections on the subspace $G_{p} X$ which has finite multiplicity. The operator $A / G_{p} X$ commutes with $\mathscr{E}_{p}$, hence, by Lemma 1 we can find an increasing sequence of projections $\left\{E_{p, h}\right\}, E_{p, h} \in \mathscr{E}_{p}$, $h, p=1,2, \ldots$ such that

$$
\left(\bigvee_{h=1}^{\infty} E_{p, h} G_{p}\right) x=\lim _{h \rightarrow \infty} E_{p, h} G_{p} x=G_{p} x, \quad x \in X,
$$

and $A E_{p, h}$ is a spectral operator of finite type on $G_{p} X$. Then $A E_{p, h} G_{p}$ will be a spectral operator of finite type on the whole space $X$. Put

$$
F_{s, h}=\bigvee_{p=1}^{s} E_{p, h} G_{p}, \quad h, s=1,2, \ldots
$$

It is easy to see that $F_{s, h} \in \mathscr{E}$ and $A F_{s, h}$ is still a spectral operator of finite type since $F_{s, h} X$ may be considered as a direct sum of invariant closed subspaces on which $A$ is a spectral operator of finite type (see Theorem C). Further, let us remark that $\left\{F_{s, h}\right\}$ is an increasing net since $s^{\prime} \leqq s^{\prime \prime}$ and $h^{\prime} \leqq h^{\prime \prime}$ imply $F_{s^{\prime}, h^{\prime}} \leqq F_{s^{\prime \prime}, h^{\prime \prime}}$. But by (3.2)

$$
\bigvee_{(s, h)} F_{s, h} \geqq \bigvee_{n=1}^{\infty} F_{r, h} \geqq \bigvee_{h=1}^{\infty} E_{r, h} G_{r}=G_{r}, \quad r=1,2, \ldots ;
$$

and consequently

$$
I \geqq \bigvee_{(s, h)} F_{s, h} \geqq \bigvee_{r=1}^{\infty} G_{r}=I
$$

Thus, by Bade [2, Lemma 2.3] the supremum and the strong limit of the net $\left\{F_{s, h}\right\}$. are the same and we shall get $\lim _{(s, h)} F_{s, h} x=x, x \in X$. Denote $P_{k}=F_{k, k}$, 
$k=1,2, \ldots$ Then $P_{k} \in \mathscr{E}, A P_{k}$ is a spectral operator of finite type, $P_{k}$ has finite multiplicity $\left(m\left(P_{k}\right) \leqq m\left(G_{k}\right)\right)$ and

$$
\bigvee_{k=1}^{\infty} P_{k}=\bigvee_{k=1}^{\infty} F_{k, k} \geqq F_{k, k} \geqq F_{s, h}, \quad k=1,2, \ldots, \quad s, h \leqq k .
$$

Therefore $\bigvee_{k=1}^{\infty} P_{k}=I$ which is equivalent with $\lim _{k \rightarrow \infty} P_{k} x=x, x \in X$. Q.E.D.

In view of the previous theorem it will be interesting to study the connection between the spectrum of an operator $A \in \Xi$ and the spectra of spectral operators $A P_{k}$ defined in Theorem 2. Since this problem may have other applications we shall study it in a more general context.

In what follows in this section we shall suppose that $\left\{P_{n}\right\}, P_{n} \neq I, n=1,2, \ldots$, is a sequence of projections converging strongly to the identity $I$ and $T$ is an operator which commutes with $P_{n}, n=1,2, \ldots$

Lemma 3. $\bigcup_{n=1}^{\infty} \sigma_{p}\left(P_{n} T\right)=\sigma_{p}(T) \cup\{0\}$.

Proof. To every $\lambda \in \sigma_{p}(T)$ there is a vector $x_{0} \neq 0$ such that $(\lambda-T) x_{0}=0$. Thus $\left(\lambda-P_{n} T\right) P_{n} x_{0}=0, n=1,2, \ldots$, where $P_{n} x_{0} \neq 0$ for $n$ sufficiently large. Consequently, $\lambda \in \sigma_{p}\left(P_{n} T\right)$ for $n$ sufficiently large. Conversely, suppose that $0 \neq \lambda \in \sigma_{p}\left(P_{n} T\right)$ for some $n$. Then, we can find $x_{0} \neq 0$ such that $\lambda x_{0}=P_{n} T x_{0}$ and, by applying $P_{n}$, we shall get $P_{n} x_{0}=x_{0}$. Hence $(\lambda-T) x_{0}=0$. Q.E.D.

Lemma 4. $\sigma_{r}(T) \subseteq\{0\} \cup \bigcup_{n=1}^{\infty} \sigma_{r}\left(P_{n} T\right)$.

Proof. If $0 \neq \lambda_{0} \in \sigma_{r}(T)$ then, $\lambda_{0}-T$ is one-to-one and by Lemma 3, $\lambda_{0}-P_{n} T$ is one-to-one also for $n=1,2, \ldots$ Therefore,

$$
\lambda_{0} \in \rho\left(P_{n} T\right) \cup \sigma_{c}\left(P_{n} T\right) \cup \sigma_{r}\left(P_{n} T\right), \quad n=1,2, \ldots
$$

If $\lambda_{0} \notin \sigma_{r}\left(P_{n} T\right), n=1,2, \ldots$, then $\left\{\left(\lambda_{0}-P_{n} T\right) X\right\}^{-}=X, n=1,2, \ldots$ Since $\lambda_{0}$ belongs to the residual spectrum of $T$ we can find a vector $y \in X-\left\{\left(\lambda_{0}-T\right) X\right\}^{-}$and a sequence $\left\{x_{n}\right\}$ such that

$$
y=\lim _{n \rightarrow \infty}\left(\lambda_{0}-P_{n} T\right) x_{n} .
$$

Relying on the fact that $\left\{\left\|P_{n}\right\|\right\}$ is a bounded sequence and $\left\{P_{n}\right\}$ converges strongly to the identity we shall get

$$
y=\lim _{n \rightarrow \infty} P_{n}\left(\lambda_{0}-P_{n} T\right) x_{n}=\lim _{n \rightarrow \infty}\left(\lambda_{0}-T\right) P_{n} x_{n},
$$

i.e., $y \in\left\{\left(\lambda_{0}-T\right) X\right\}^{-}$which contradicts the choice of $y$. Thus $\lambda_{0}$ belongs to the residual spectrum of $P_{n} T$ for some $n$. Q.E.D.

Foguel has proved in $[8$, Theorem 1, p. 56] that a spectral operator of finite type has not residual spectrum. From this theorem, Lemma 4 and Theorem 2 we can get the next result.

Corollary 5. If $A \in \Xi, \sigma_{r}(A) \subseteq\{0\}$. 
LEMMA 5. If $P_{n} T, n=1,2, \ldots$, are spectral operators of the same type $m$ and their resolutions of the identity are uniformly bounded

$$
\left\|E\left(P_{n} T, \cdot\right)\right\| \leqq M_{1}, \quad n=1,2, \ldots,
$$

then

$$
\sigma(T) \cup\{0\}=\left\{\bigcup_{n=1}^{\infty} \sigma\left(P_{n} T\right)\right\}^{-} .
$$

Proof. As a consequence of Gelfand's representation theory of commutative $B$-algebras

$$
\sigma\left(P_{n} T\right) \subseteq \sigma\left(P_{n}\right) \cdot \sigma(T)=\{0\} \cup \sigma(T), \quad n=1,2, \ldots
$$

Then,

$$
\left\{\bigcup_{n=1}^{\infty} \sigma\left(P_{n} T\right)\right\}^{-} \subseteq\{0\} \cup \sigma(T) .
$$

Conversely, let $0 \neq \lambda_{0} \in \sigma(T)$; if $\lambda_{0} \in \sigma_{p}(T) \cup \sigma_{r}(T)$ then, the conclusion follows immediately from Lemmas 3 and 4. Hence, we can assume that $\lambda_{0} \in \sigma_{c}(T)$ and $\lambda_{0} \notin\left\{\bigcup_{n=1}^{\infty} \sigma\left(P_{n} T\right)\right\}^{-}$. Now, let $W$ be an open circle around $\lambda_{0}$ of radius $r ; 0<r<1$ such that

$$
W \cap\left(\bigcup_{n=1}^{\infty} \sigma\left(P_{n} T\right)\right)=\varnothing .
$$

Using Theorem B for the spectral operator $P_{n} T$ (of type $m$ ) we shall get

$$
R\left(\lambda_{0}, P_{n} T\right)=\sum_{k=0}^{m} N_{n}^{k} \int_{\sigma\left(P_{n} T\right)} \frac{E\left(P_{n} T, d \mu\right)}{\left(\lambda_{0}-\mu\right)^{k+1}}, \quad n=1,2, \ldots,
$$

where $N_{n}$ is the nilpotent part of $P_{n} T$. Thus

$$
\left\|R\left(\lambda_{0}, P_{n} T\right)\right\| \leqq \sum_{k=0}^{m}\left\|N_{n}^{k}\right\| \frac{4 M_{1}}{r^{k+1}} \leqq \frac{4 M_{1}}{r^{m+1}} \sum_{k=0}^{m}\left\|N_{n}^{k}\right\|, \quad n=1,2, \ldots
$$

Since $\left\{P_{n}\right\}$ converges strongly to $I$ there exists a constant $M_{2}$ such that $\left\|P_{n}\right\| \leqq M_{2}$, $n=1,2, \ldots$ Then, if $S_{n}$ denotes the scalar part of $P_{n} T$, we get

$$
\begin{aligned}
\left\|N_{n}\right\| \leqq\left\|P_{n} T\right\|+\left\|S_{n}\right\| & \leqq M_{2}\|T\|+4 M_{1} \sup _{\lambda \in \sigma\left(P_{n} T\right)}|\lambda| \\
& \leqq M_{2}\|T\|+4 M_{1} \sup _{\lambda \in \sigma(T)}|\lambda|=M_{3},
\end{aligned}
$$

where $M_{3}$ does not depend on $n$. With no loss of generality we can suppose that $M_{3} \geqq 1$. Hence,

$$
\left\|R\left(\lambda_{0}, P_{n} T\right)\right\| \leqq 4 m M_{1} M_{3}^{m} / r^{m+1}=M_{4}, \quad n=1,2, \ldots,
$$

where $M_{4}$ also does not depend on $n$. But

$$
\inf _{\| x i !=1}\left\|\left(\lambda_{0}-P_{n} T\right) x\right\|=1 /\left\|R\left(\lambda_{0}, P_{n} T\right)\right\| \geqq 1 / M_{4}, \quad n=1,2, \ldots ;
$$


therefore

$$
\left\|\left(\lambda_{0}-T\right) x\right\| \geqq 1 / M_{4}, \quad x \in X, \quad\|x\|=1,
$$

and consequently $\lambda_{0} \notin \sigma(T)$ which contradicts the hypotheses. Q.E.D.

It will be seen in a following example (in \$5) that we may not prove the previous lemma without the assumption that $P_{n} T, n=1,2, \ldots$ are spectral operators of the same finite type. The next necessary and sufficient condition for spectrality will be useful in what follows.

THEOREM 7. Assume that:

(i) $P_{k} P_{n}=P_{n} P_{k}=P_{k}$ for $k \leqq n$,

(ii) $P_{n} T=S_{n}+N_{n}, n=1,2, \ldots$, are spectral operators of the same finite type $m$ and $S_{n}$ and $N_{n}$ the respective scalar and nilpotent parts.

Then $T$ is spectral (of type $m$ ) if and only if there is a constant $M$ such that

$$
\left\|E\left(P_{n} T, \delta\right)\right\| \leqq M, \quad n=1,2, \ldots,
$$

for every Borel set $\delta$. Furthermore,

$$
E(T, \delta) x=\lim _{n \rightarrow \infty} E\left(P_{n} T, \delta\right) x, \quad x \in X, \quad \delta \in \text { Borel sets. }
$$

Proof. Let $T$ be spectral and assume $\left\|P_{n}\right\| \leqq K_{1}, n=1,2, \ldots$, and $\|E(T, \delta)\| \leqq K_{2}$, $\delta \in$ Borel sets. Then $T / P_{n} X, n=1,2, \ldots$, are spectral and their resolutions of the identity are uniformly bounded by $K_{1} K_{2}$ and hence (3.9) holds for $M=K_{1} K_{2}$ $+\left(1+K_{1}\right)$. Now, suppose that (3.9) is satisfied. If $k<n, P_{k} T=P_{k} P_{n} T$, thus, $P_{n} T / P_{k} X=P_{k} T / P_{k} X$. Hence, their resolutions of the identity coincide (on the invariant subspace $\left.P_{k} X\right)$ i.e., $E\left(P_{n} T, \cdot\right) P_{k}=E\left(P_{k} T, \cdot\right) P_{k}$. Take $x \in \bigcup_{n=1}^{\infty} P_{n} X$ and let $k$ be the first integer for which $x \in P_{k} X$. Then, for $n>k$,

$$
E\left(P_{n} T, \cdot\right) x=E\left(P_{n} T, \cdot\right) P_{k} x=E\left(P_{k} T, \cdot\right) P_{k} x=E\left(P_{k} T, \cdot\right) x
$$

and, consequently, we can define the operator

$$
E(\cdot) x=\lim _{n \rightarrow \infty} E\left(P_{n} T, \cdot\right) x, \quad x \in \bigcup_{n=1}^{\infty} P_{n} X .
$$

Since the set $\bigcup_{n=1}^{\infty} P_{n} X$ is dense and $\|E(\cdot) x\| \leqq M\|x\|$ for $x \in \bigcup_{n=1}^{\infty} P_{n} X$ the operator $E(\cdot)$ can be defined in a unique way on the whole space $X$ such that it will be a bounded linear operator satisfying

$$
E(\cdot) x=\lim _{n \rightarrow \infty} E\left(P_{n} T, \cdot\right) x, \quad x \in X .
$$

Obviously, $E(\cdot)$ is a spectral measure commuting with $T$. In view of [5, Theorem IV-10-6], $E(\cdot) x$ is countable additive for every $x \in X$. By Lemma 6 (applied on $E(\delta) X, \delta \in$ Borel sets)

$$
\begin{aligned}
\sigma(T / E(\delta) X) & \subseteq\left\{\bigcup_{n=1}^{\infty} \sigma\left(P_{n} T / E(\delta) X\right)\right\}^{-} \subseteq\left\{\bigcup_{n=1}^{\infty} \sigma\left(P_{n} E(\delta) T\right)\right\}^{-} \\
& =\left\{\bigcup_{n=1}^{\infty} \sigma\left(P_{. n} E\left(P_{n} T, \delta\right) T\right)\right\}^{-} \subseteq\{0\} \cup\left\{\bigcup_{n=1}^{\infty} \sigma\left(P_{n} T / E\left(P_{n} T, \delta\right) x\right)\right\}^{-} \subseteq\{0\} \cup \delta
\end{aligned}
$$


and if $0 \notin \sigma(T)$ then $E(\cdot)$ is the resolution of the identity for $T$ and (3.10) holds. If $0 \in \sigma(T)$ we can find a scalar $\lambda \in \rho(T)$ and, further, we can prove that $\lambda-T$ is spectral (by applying the first part of the proof to it). Then, by [7, Lemma 1] $T$ is also spectral and (3.10) is satisfied. Q.E.D.

This theorem shows that, in fact, Lemma. 6 deals only with spectral operators of finite type. Now, we can improve it as follows.

THEOREM 8. Assume that $T=S+N$ is a spectral operator whose scalar and generalized nilpotent parts are $S$, respectively $N$. Then (3.6) holds.

Proof. We shall follow the proof of Lemma 6. As in it, it suffices to show that $\lambda_{0} \in \sigma_{c}(T)$ implies $\lambda_{0} \in\left\{\bigcup_{n=1}^{\infty} \sigma\left(P_{n} T\right)\right\}^{-}$. First, remark that in view of [7, Theorem 2] $P_{n} T$ are spectral operators and there is a constant $M_{1}$ such that

$$
\left\|E\left(P_{n} T, \cdot\right)\right\| \leqq M_{1}, \quad n=1,2, \ldots
$$

There also exists a constant $M_{2}$ such that $\left\|P_{n}\right\| \leqq M_{2}, n=1,2, \ldots$ If for some $\lambda_{0} \in \sigma_{c}(T)$ we have $\lambda_{0} \notin\left\{\bigcup_{n=1}^{\infty} \sigma\left(P_{n} T\right)\right\}^{-}$, then, following the idea of the proof of Lemma 6 we get

$$
\left\|R\left(\lambda_{0}, P_{n} T\right)\right\| \leqq 4 M_{1} \sum_{k=0}^{\infty} \frac{\left\|N_{n}^{k}\right\|}{r^{k+1}} \leqq \frac{4 M_{1} M_{2}}{r} \sum_{k=0}^{\infty} \frac{\left\|N^{k}\right\|}{r^{k}}
$$

since the generalized nilpotent part of $P_{n} T$ satisfies $N_{n}=P_{n} N, n=1,2, \ldots$ If $\left\|R\left(\lambda_{0}, P_{n} T\right)\right\|$ is uniformly bounded for $n=1,2, \ldots$ we finish the proof as in Lemma 6, if not, we can assume the existence of a subsequence $\left\{R\left(\lambda_{0}, P_{n_{1}} T\right)\right\}$ for which $\lim _{i \rightarrow \infty}\left\|R\left(\lambda_{0}, P_{n_{1}} T\right)\right\|=\infty$. Consequently $\sum_{k=1}^{\infty}\left\|N^{k}\right\| / r^{k}$ diverges and $\left(\left\|N^{k}\right\| / r^{k}\right)^{1 / k}>\frac{1}{2}$ for $k$ sufficiently large; i.e., $N$ is not a generalized nilpotent as we have assumed. Q.E.D.

4. Strong limits in $\Xi$. The author has proved in [12] (see also Theorem E) that a strong limit of spectral operators commuting with a complete countably decomposable B.A. of projections of finite uniform multiplicity is spectral provided their resolutions of the identity are uniformly bounded. Now, we shall generalize this theorem under the additional assumption that the underlying space is a separable Hilbert space.

Lemma 9. Let $T$ be a spectral operator in a separable Banach space $X$ and $G(\cdot)$ its resolution of the identity. If $\|G(\delta)\| \leqq M$ for every Borel set $\delta$ for which $G(\partial \delta)=0$, then,

$$
\|G(\sigma)\| \leqq M, \quad \sigma \in \text { Borel sets. }
$$

Proof. Let $\eta$ be a closed set in the complex plane. Define

$$
\eta_{r}=\{\mu|| \mu-\lambda \mid<r \text { for some } \lambda \in \eta), \quad 0<r \leqq 1 .
$$

These open sets $\eta_{r}$ satisfy:

$$
\eta_{r^{\prime}} \subseteq \bar{\eta}_{r^{\prime}} \subseteq \eta_{r^{\prime \prime}}, \quad 0<r^{\prime}<r^{\prime \prime} \leqq 1
$$


Now, let $\left\{x_{n}\right\}$ be a dense sequence. By [2, Theorem 3.1], to every vector $x_{n}$ there is a linear functional $x_{n}^{*} \in X^{*}$ with the properties:

(i) $x_{n}^{*} G(\delta) x_{n} \geqq 0, \delta \in$ Borel sets,

(ii) if $x_{n}^{*} G(\delta) x_{n}=0$, then $G(\delta) x_{n}=0$.

Denote

$$
g_{n}(r)=x_{n}^{*} G\left(\eta_{r}\right) x_{n}, \quad 0<r \leqq 1, \quad n=1,2, \ldots
$$

In view of (4.2) $g_{n}(r)$ is an increasing function of $r$, hence, the set $D_{n}$ of all its points of discontinuity is at most countable, consequently, the set $D=\bigcup_{n=1}^{\infty} D_{n}$ is also at most countable. But

$$
x_{n}^{*} G\left(\eta_{r_{0}}\right) x_{n} \leqq x_{n}^{*} G\left(\bar{\eta}_{r_{0}}\right) x_{n} \leqq x_{n}^{*} G\left(\eta_{r}\right) x_{n}, \quad r_{0}<r,
$$

and, for $r_{0} \notin D, \lim _{r \rightarrow r_{0}} g_{n}(r)=g_{n}\left(r_{0}\right), n=1,2, \ldots$; therefore

$$
x_{n}^{*} G\left(\eta_{r_{0}}\right) x_{n}=x_{n}^{*} G\left(\bar{\eta}_{r_{0}}\right) x_{n}, \quad n=1,2, \ldots
$$

Relying on the properties of $x_{n}^{*}$ we shall get $G\left(\eta_{r_{0}}\right) x_{n}=G\left(\bar{\eta}_{r_{0}}\right) x_{n}, n=1,2, \ldots, r_{0} \notin D$ and further $G\left(\eta_{r_{0}}\right)=G\left(\bar{\eta}_{r_{0}}\right), r_{0} \notin D$. In conclusion $G\left(\partial \eta_{r_{0}}\right)=0, r_{0} \notin D$, i.e.,

$$
\left\|G\left(\eta_{r_{0}}\right)\right\| \leqq M, \quad r_{0} \notin D .
$$

Now, let us choose a decreasing sequence $\left\{r_{j}\right\}$ such that $r_{j} \notin D$ and $\lim _{j \rightarrow \infty} r_{j}=0$. Then by [2, Lemma 2.3] we shall obtain

$$
G(\eta) x=G\left(\bigcap_{j=1}^{\infty} \eta_{r_{j}}\right) x=\left(\bigwedge_{j=1}^{\infty} G\left(\eta_{r_{j}}\right)\right) x=\lim _{j \rightarrow \infty} G\left(\eta_{r_{j}}\right) x, \quad x \in X .
$$

Hence $\|G(\eta)\| \leqq M$ for every closed set $\eta$. Since $x^{*} G(\cdot) x, x \in X, x^{*} \in X^{*}$, is a regular measure we can conclude that (4.1) holds also. Q.E.D.

Lemma 10. Let $X$ be a separable Hilbert space and $\left\{T_{n}\right\}$ a sequence of spectral operators converging strongly on $X$ to a spectral operator $T$. If the resolutions of the identity of $T_{n}, n=1,2, \ldots$ satisfy

$$
\left\|G\left(T_{n}, \delta\right)\right\| \leqq K, \quad n=1,2, \ldots, \quad \delta \in \text { Borel sets, }
$$

and the sequence of the scalar parts of $T_{n}$ converges strongly to the scalar part of $T$ and the same assertion holds with respect to the generalized nilpotent parts, real parts and imaginary parts, then the resolution of the identity of $T$ satisfies

$$
\|G(T, \delta)\| \leqq 4 K^{3}, \quad \delta \in \text { Borel sets. }
$$

Proof. Let $T_{n}=S_{n}+N_{n}, T=S+N$ be their decomposition as a sum of a spectral operator of scalar type and a generalized nilpotent one. If $R_{n}, R$ and $J_{n}, J$ are the real, respectively imaginary parts of $S_{n}, S$, then by [8, Lemma 1, p. 59] their resolutions of the identity satisfy

$$
\left\|G\left(R_{n}, \delta\right)\right\| \leqq K, \quad\left\|G\left(J_{n}, \delta\right)\right\| \leqq K, \quad n=1,2, \ldots, \quad \delta \in \text { Borel sets. }
$$

By [1, Theorem 2.6] $\|G(R, \delta)\| \leqq K$ for every Borel set $\delta$ for which $G(R, \partial \delta)=0$ and the same for $G(J, \delta)$. Thus, by Lemma 9 ,

$$
\|G(R, \sigma)\| \leqq K, \quad\|G(J, \sigma)\| \leqq K, \quad \sigma \in \text { Borel sets. }
$$


By using Theorem $\mathrm{D}$, since $S=R+i J$, we shall get

$$
\|G(S, \sigma)\|=\|G(T, \sigma)\| \leqq 4 K^{3}
$$

for every Borel set $\sigma$. Q.E.D.

LeMma 11. Let $\mathscr{B}$ be a complete countably decomposable B.A. of projections of finite multiplicity $p$ on a separable Banach space $X$ and $\left\{T_{n}\right\}$ a sequence of spectral operators commuting with $\mathscr{B}$ and converging strongly to an operator $T$. Let $G\left(T_{n}, \cdot\right)$ denote the resolution of the identity for $T_{n}$, if there is a constant $M$ such that $\left\|G\left(T_{n}, \delta\right)\right\| \leqq M, n=1,2, \ldots, \delta \in$ Borel sets, then $T$ is spectral (of type $p$ ). Moreover, the sequence of the scalar parts of $T_{n}$ converges strongly to the scalar part of $T$ and the same assertion holds with respect to the nilpotent parts, real parts and imaginary parts.

Proof. By (1.4) $I=\bigvee_{j=1}^{p} E_{j}=\sum_{j=1}^{p} E_{j}$ where $E_{j}, j=1,2, \ldots, p$, are disjoint projections belonging to $\mathscr{B}, E_{j}=0$ or it has uniform multiplicity $j$. Denote

$$
\mathscr{B}_{j}=\left\{E / E_{j} X \mid E \in \mathscr{B}\right\} .
$$

Then $\mathscr{B}_{j}$ is a complete countably decomposable (since $X$ is separable) B.A. of projections on $E_{j} X$ and it has uniform multiplicity $j$ (if $E_{j} \neq 0$ ). Remark that $T_{n} / E_{j} X, n=1,2, \ldots$, are spectral operators which commute with $\mathscr{B}_{j}$ and their resolutions of the identity are uniformly bounded. Then, by Theorem $E, T / E_{j} X$ are spectral and further, by Theorem C, $T$ is also spectral (of type $p$ ). The last statement of this theorem follows from the similar assertion proved in Theorem E. Q.E.D.

THEOREM 12. Let $X$ be a separable Hilbert space and $A_{n} \in \Xi, n=1,2, \ldots, a$ sequence of spectral operators of the same finite type $p$ converging strongly to an operator A. Let $G\left(A_{n}, \cdot\right)$ denote the resolution of the identity for $A_{n}$, if there is a constant $M$ such that $\left\|G\left(A_{n}, \delta\right)\right\| \leqq M, n=1,2, \ldots, \delta \in$ Borel sets, then $A$ is spectral (of type p). Moreover, the sequence of the scalar parts of $A_{n}$ converges strongly to the scalar part of $A$ and the same assertion holds with respect to the nilpotent parts, real parts and imaginary parts.

Proof. By Theorem 2 applied to $A$, there is a sequence of projections $\left\{P_{k}\right\}$, $P_{k} \in \mathscr{E}, k=1,2, \ldots$, converging strongly to the identity, increasing and such that $A P_{k}$ is a spectral operator of finite type. Furthermore, $P_{k}$ has finite multiplicity for $k=1,2, \ldots$. Assume that $\left\|P_{k}\right\| \leqq L, k=1,2, \ldots$ and denote

$$
\mathscr{E}_{k}=\left\{E\left|P_{k} X\right| E \in \mathscr{E}\right\}, \quad k=1,2, \ldots
$$

Then $\mathscr{E}_{k}, k=1,2, \ldots$, are complete countably decomposable B.A. of projections of finite multiplicity. The operators $A_{n} / P_{k} X, n=1,2, \ldots$, commute with $\mathscr{E}_{k}$ and their resolutions of the identity satisfy

$$
\left\|G\left(A_{n} / P_{k} X, \delta\right)\right\| \leqq M L, \quad n, k=1,2, \ldots, \quad \delta \in \text { Borel sets. }
$$


Thus, by Lemma $11 A / P_{k} X, k=1,2, \ldots$, are spectral operators of type $p$ and by Lemma 10 their resolutions of the identity satisfy

$$
\left\|G\left(A \mid P_{k} X, \delta\right)\right\| \leqq 4(M L)^{3}, \quad k=1,2, \ldots, \quad \delta \in \text { Borel sets. }
$$

Consequently, $A P_{k}, k=1,2, \ldots$ are spectral operators of type $p$ and their resolutions of the identity are uniformly bounded. Then, by Theorem 7, $A$ is spectral (of type $p$ ).

If $S_{n}, S$ denote the respective scalar parts of $A_{n}$ and $A$, then it is easy to see that

$$
\lim _{n \rightarrow \infty} S_{n} x=S x, \quad x \in \bigcup_{k=1}^{\infty} P_{k} X .
$$

But this union is a dense set and $\left\{\left\|S_{n}\right\|\right\}$ is a bounded sequence as a consequence of the uniform boundedness of $G\left(A_{n}, \cdot\right)$; hence $\left\{S_{n}\right\}$ converges strongly to $S$. Thus, the sequence of the nilpotent parts of $A_{n}$ converges strongly to the nilpotent part of $A$. Similar arguments for the real and imaginary parts will finish the proof. Q.E.D.

5. Examples. The following examples are designed to show that some conditions imposed in Lemma 6, Theorem 7 and Theorem 12 are necessary.

Let $\left\{e_{k}\right\}$ be the usual basis of the space $l_{2}$. To every $x=\sum_{k=1}^{\infty} x_{k} e_{k} \in l_{2}$ let us define

$$
\begin{aligned}
U x= & 0 e_{1} \\
& +0 e_{2}+x_{2} e_{3} \\
& +0 e_{4}+x_{4} e_{5}+x_{5} e_{6} \\
& +0 e_{7}+x_{7} e_{8}+x_{8} e_{9}+x_{9} e_{10} \\
& +. \quad . \quad . \quad . \quad . \quad .
\end{aligned}
$$

Obviously, $U$ is a bounded linear operator in $l_{2},\|U\|=1$ and its spectral radius is equal to 1 , i.e., $U$ is not a generalized nilpotent. Now, let $Q_{n}, n=1,2, \ldots$, be the orthogonal projection on the subspace generated by $\left\{e_{1}, e_{2}, \ldots, e_{n}\right\}$. The operator $U$ commutes with $Q_{1}, Q_{3}, Q_{6}, Q_{10}, \ldots$ and

$$
\begin{aligned}
& U Q_{1} x=0 \\
& U Q_{3} x=x_{2} e_{3} \\
& U Q_{6} x=x_{2} e_{3}+x_{4} e_{5}+x_{5} e_{6} \\
& U Q_{10} x=x_{2} e_{3}+x_{4} e_{5}+x_{5} e_{6}+x_{7} e_{8}+x_{8} e_{9}+x_{9} e_{10}
\end{aligned}
$$

hence $U Q_{1}$ is a nilpotent of order $1, U Q_{3}$ of order $2, U Q_{6}$ of order $3, U Q_{10}$ of order 4 and so forth. Since $\left\{Q_{1}, Q_{3}, Q_{6}, Q_{10}, \ldots\right\}$ converges strongly to the identity and the resolutions of the identity of $U Q_{1}, U Q_{3}, U Q_{6}, U Q_{10}, \ldots$ are uniformly bounded by 1 we have an example which shows that in Lemma 6 the operators $T P_{n}$, $n=1,2, \ldots$, have to be of the same finite type. The same restriction on the type of 
$T P_{n}, n=1,2, \ldots$, is necessary in order to prove Theorem 7 for, if $U$ is spectral, then by Theorem 8 it must be a generalized nilpotent.

Now, let $\mathscr{F}$ be the complete B.A. of projections generated by

$$
\left\{Q_{1}, Q_{3}, Q_{6}, Q_{10}, \ldots\right\}
$$

(the existence of a complete B.A. of projections is insured by [1, Theorem 3.2], since $l_{2}$ is reflexive). Observe that $Q_{1}, Q_{3}, Q_{6}, Q_{10}, \ldots$ have finite multiplicity and

$$
I=Q_{1} \vee Q_{3} \vee Q_{6} \vee Q_{10} \vee \cdots .
$$

Hence $\mathscr{F}$ contains no projections of infinite uniform multiplicity and $U$ commutes with $\mathscr{F}$. Since the resolutions of the identity for $U Q_{1}, U Q_{3}, U Q_{6}, U Q_{10}, \ldots$ are uniformly bounded we get an example which justifies why in Theorem 12 the operators $A_{n}, n=1,2, \ldots$, are required to be of the same finite type.

Added in proof. Meanwhile, we have observed that the main Theorem 12 remains true in separable Banach spaces. Indeed, let us remark that the assumption that underlying space is a Hilbert space was used only through Lemma 10 and can be replaced now in the proof of Theorem 12 by Corollary 2 of L. Tzafriri, Perturbations of spectral operators, Israel J. Math. 4 (1966), 62-64.

\section{REFERENCES}

1. W. G. Bade, Weak and strong limits of spectral operators, Pacific J. Math. 4 (1954), 393-413.

2. - On Boolean algebras of projections and algebras of operators, Trans. Amer. Math. Soc. 80 (1955), 345-359.

3. - A multiplicity theory for Boolean algebras of projections on Banach spaces, Trans. Amer. Math. Soc. 92 (1959), 508-530.

4. N. Dunford, Spectral operators, Pacific J. Math. 4 (1954), 321-354.

5. N. Dunford and J. Schwartz, Linear operators. I, Interscience, New York, 1958.

6. N. Dunford, A spectral theory for certain operators on a direct sum for Hilbert spaces, Math. Ann. 162 (1966), 294-330.

7. S. R. Foguel, Sums and products of commuting spectral operators, Ark. Mat. 3 (1957), 449-461.

8. - The relations between a spectral operator and its scalar part, Pacific J. Math. 8 (1958), 51-65.

9. — Normal operators of finite multiplicity, Comm. Pure Appl. Math. 11 (1958), 297-313.

10. - Boolean algebras of projections of finite multiplicity, Pacific J. Math. 9 (1959), 681-693.

11. C. A. McCarthy, Commuting Boolean algebras of projections, Pacific J. Math. 11 (1961), 295-307.

12. L. Tzafriri, Operators commuting with Boolean algebras of projections of finite multiplicity, Pacific J. Math. (to appear).

13. J. Wermer, Commuting spectral measures on Hilbert space, Pacific J. Math. 4 (1954), 355-361.

The Hebrew University, JERUSALEM, ISRAEL 